\title{
Mix-and-Match Compatibility in Asymmetric System Markets
}

\author{
by
}

Jong-Hee Hahn and Sang-Hyun Kim*

Received November 25, 2009; in revised form June 29, 2011; accepted June 29, 2011

\begin{abstract}
This paper shows that the private incentive for mix-and-match compatibility in system markets diverges from the social planner's incentive if competing suppliers are asymmetric in production cost or product quality. There can be too much or too little compatibility when the market is served by fully integrated system suppliers. Also, the market outcome involves socially too much incompatibility in the form of exclusive technological alliances when the market is composed of independent component suppliers. These results contrast with the standard one obtained in the symmetric setup and shed new light on public policy towards compatibility, technological alliances, and bundling practices in system markets. (JEL: D42, L12)
\end{abstract}

\section{Introduction}

Many goods are used in systems composed of several complementary components. Examples are widespread: bolts and nuts, which together provide fastening; computer hardware and software, which provide information processing; network platforms and contents, which provide various information/entertainment services. In such a market, an issue that is important not only for business strategy but also for public policy is whether to make components produced by different firms compatible or make them incompatible with each other. If components are incompatible, the competition is system versus system and consumers have no choice but to buy

* Jong-Hee Hahn (corresponding author) is at Yonsei University, Seoul, and SangHyun Kim is at Michigan State University, East Lansing. We would like to thank Elmar Wolfstetter and two anonymous referees for insightful comments and suggestions. This work was supported by a Korea Research Foundation Grant funded by the Korean Government (NRF-2008-2-B00135). Jong-Hee Hahn acknowledges financial support from the National Research Foundation of Korea funded by the Ministry of Education, Science and Technology through its World Class University Grant (R322008-000-10056-0). This work was supported by the National Research Foundation of Korea Grant funded by the Korean Government (NRF-2010-330-B0092).

Journal of Institutional and Theoretical Economics

JITE 168 (2012), 311-338 @ 2012 Mohr Siebeck - ISSN 0932-4569 
a system whose components are provided by a single firm. If components are compatible, consumers can mix and match their preferred components to build their best system.

Matutes and Regibeau (1988) and Economides (1989), hereafter MR\&E, were the first to analyze this kind of mix-and-match compatibility in system markets with horizontally differentiated components. They examine firms' incentive for compatibility and its welfare implication. The main conclusion of their analyses is that compatibility is beneficial to the firms as well as to the society. Compatibility softens price competition, since a firm cannot internalize the full benefit of its price cut with compatible components; and it also enhances social welfare by increasing the variety of systems in the market. See also Einhorn (1992) for a similar analysis in the context of vertically differentiated markets, where compatibility softens competition by increasing the degree of quality differentiation. Matutes and Regibeau (1992) allow firms to price a bundle of their components separately from individual components. Boom (2001) analyzes firms' product-characteristic choices prior to compatibility decisions. These results imply that the private market incentive coincides with the social incentive and therefore public policy need not intervene in firms' decisions on compatibility.

Given that MR\&E's analyses are restricted to the case of symmetric firms, it would be interesting to see whether their result still holds when competing firms are asymmetric in production cost or product quality. In fact, most, if not all, system markets in the real world are characterized by competition among asymmetric firms with unequal market shares. We can easily find examples where asymmetric firms with different market shares compete with compatible components, such as the present hi-fi and electric appliance industries. There also exist examples of asymmetric firms competing with incompatible components, such as the digital camera industry, where some memory cards are compatible only with certain types of digital cameras. For example, the xD-Picture Card, developed by Olympus and FujiFilm, is used only for digital cameras produced by the two firms, and the Memory Stick, developed by Sony, is used only for its own digital cameras. Note that, however, some memory cards, such as the Compact Flash Card, are compatible with digital cameras produced by many different manufacturers.

This paper examines how the firms' incentive for compatibility and its welfare effect are affected by the presence of asymmetry in system markets. Analyzing a stylized model built on Matutes and Regibeau (1988), we find that, contrary to MR\&E's result obtained in the symmetric setup, the market incentive for compatibility may diverge from the social optimum if one firm has sufficiently large cost advantages over all components. First, for a moderate level of asymmetry there can be excess compatibility in the market. ${ }^{1}$ The firms opt for compatibility with higher equilibrium prices, as in the symmetric case. Compatibility, however, involves too

1 A similar point has been noted by Matutes and Regibeau (1988) and Boom (2001). However, their results critically depend on the nonnegligible cost of attaining compatibility. Indeed, ignoring the cost of compatibility, their models yield that compatibility is always (weakly) welfare-improving. Assuming away the cost of attaining 
much inefficient production by the high-cost firm, in comparison with incompatibility. This is because the inefficient firm's market share tends to be greater under compatibility, where cost advantages or disadvantages between the firms are split over components and therefore their effect on the market shares is weaker than in the case of incompatibility, and also because price competition is less intense under compatibility with imperfect appropriation of the price-cutting benefit. This welfare loss from inefficient production outweighs the gain from increased product variety under compatibility. Second, for a high level of asymmetry the market outcome may involve socially too much incompatibility. Here, the intensity of price competition is lower under incompatibility rather than compatibility. This is because the market boundary is shorter under incompatibility than under compatibility, and so the firms' price-cutting incentives are lower under incompatibility. This market-boundary effect dominates the imperfect-appropriation effect in this case, and therefore the firms prefer incompatibility over compatibility. Social welfare, however, is larger under compatibility, with more efficient production as well as more diverse systems. Note that the latter result depends on a specific assumption on the distribution of consumer preferences, while the former result is quite robust to model specification. This point will be discussed in more detail later, in section 6 . If cost advantages are shared between the firms across components, on the other hand, the firms prefer compatibility and it is socially desirable as well, exactly the same as the standard result with symmetric firms. Here, compatibility increases firm profits by relaxing price competition due to the imperfect-appropriation effect, and also improves social welfare by allowing the firms to specialize in components they can produce more efficiently.

We also examine the case where the market is served by several independent component suppliers. As noted by Matutes and Regibeau (1988, p. 228), with separate ownership across components there is no difference in the degree of internalization of complementarity between compatibility and incompatibility, and so (symmetric) firms are indifferent between the two regimes. With asymmetric firms, however, we find that the market outcome leads to too much incompatibility. Efficient complementary suppliers have joint incentives to enforce compatibility only between their components and not with those produced by other, inefficient firms; this decision may be implemented by means of an exclusive technological alliance. In fact, inefficient firms also benefit from incompatibility. This is mainly because incompatibility relaxes the intensity of price competition by reducing the equilibrium market boundary. Incompatibility, however, is detrimental to social welfare, since it does not allow consumers to mix and match their preferred components, and also softer price competition under incompatibility allows inefficient firms to produce more than under compatibility.

Work on mix-and-match compatibility in asymmetric system markets is rather scant in the literature. Farrell, Monroe, and Saloner (1998) show that incompatibility

compatibility, our result gives a sharper and direct welfare comparison between compatibility and incompatibility. 
may prevail in the market with more than two varieties of each component and cost heterogeneity across the firms. Choi (1996) examines the relation between compatibility and R\&D incentives in a mix-and-match model with cost asymmetry. ${ }^{2}$ Assuming identical components or homogeneous consumer preferences, however, their analyses focus only on the effect compatibility or incompatibility has on the cost of production or the level of $\mathrm{R} \& \mathrm{D}$, failing to capture its effect on system variety and consumer surplus. Using a mix-and-match model with differentiated products, we show clearly how the variety-enhancing effect interacts with the cost effect when the competing firms are asymmetric, and how the model's policy implications differ from those obtained in the early literature.

The rest of the paper is structured as follows. Section 2 presents the model. Sections 3 and 4 characterize equilibria under compatibility and incompatibility and derive the main results when the market is served by integrated system suppliers. In section 5 we extend the analysis to the case of independent component suppliers with separate ownership. Section 6 discusses the robustness of the results. Section 7 concludes with discussions on policy implications regarding compatibility, bundling, and entry deterrence in system markets.

\section{The Model}

The model is essentially the same as the one in Matutes and Regibeau (1988), except for asymmetries in production cost across firms. ${ }^{3}$ Consider a market consisting of two integrated system suppliers and a continuum of consumers. Each firm produces two complementary components, $A$ and $B$, that are used on a one-to-one basis to form systems. Assume that the two firms produce both components at constant marginal costs without fixed costs. The firms' products are horizontally differentiated. Following Matutes and Regibeau (1988), we assume that consumers of mass 1 are uniformly distributed on the unit square, where firm 1 is located at the origin and firm 2 at the point of coordinates $(1,1) .{ }^{4}$ Consumers have unit demands for the system, and a consumer's location on the square denotes her most preferred specification of the two components. The net surplus of a consumer who buys one

2 Also related is the paper by Denicolo (2000). He analyzes a structurally asymmetric setup with one generalist firm offering both components of a system competing against two specialists each supplying one component only, and shows that the generalist firm may have an incentive to choose incompatibility.

3 Note that representing asymmetry between firms in terms of quality rather than cost would not change the results qualitatively, provided the quality of a system is taken to be the sum of component qualities and consumers have identical preferences for quality.

4 This assumption is not totally innocuous under asymmetry, since with asymmetric firms the relative intensity of competition under compatibility and incompatibility depends on the shape of the consumer distribution. Nevertheless, it serves as a natural benchmark for the purpose of comparison, given that most of the previous works are based on the same model setup. Later we will discuss the robustness of our results with respect to this assumption. 
unit of component $A$ from firm $i$ and one unit of component $B$ from firm $j$ is given by

$$
V-\lambda\left(d_{A i}+d_{B j}\right)-P_{i j}, \quad i, j=1,2,
$$

where $V$ is the reservation price for a unit of the system (common to all consumers), $d_{z i}$ is the distance between the consumer's preferred specification of component $z$ and the specification of component $z$ sold by firm $i(i=1,2, z=A, B)$, and $P_{i j}$ is the total price the consumer pays for a system composed of firm $i$ 's component $A$ and firm $j$ 's component $B$. The parameter $\lambda$ measures the degree of horizontal differentiation between the two firms' components, which is normalized to 1 for analytical simplicity. A consumer located at the point of coordinates $\left(x_{1}, x_{2}\right)$ has her preferred component $A$ that is $x_{1}$ away from the specification of firm 1's component $A$ and her preferred component $B$ that is $x_{2}$ away from the specification of firm 1's component $B$. Similarly, the distances between the consumer's preferred components and firm 2's components are $1-x_{1}$ and $1-x_{2}$. Individual consumers will buy a unit of the system, provided they obtain a nonnegative net surplus. We assume that $V$ is sufficiently large so that the market is covered in equilibrium (i.e., every consumer makes a system). We will discuss the cases of partial participation in section 6 .

We solve for a perfect Nash equilibrium in the following two-stage game. In the first stage, each firm decides whether to make its components compatible with its rival's ones. In the second stage, the firms compete in price, given the first-stage compatibility decisions. We assume that the first-stage compatibility decisions are irreversible (or very costly to reverse) in the later stage. They may involve fundamental choices on technologies and system designs. Two cases can be considered regarding the mechanism by which compatibility is attained in the market. The first is the consensus case, where each firm's proprietary technologies are protected by intellectual-property rights and cannot be used by the rival without the rightholder's consent. In this case compatibility requires bilateral agreements between the firms to make their components compatible with each other or to adopt a common technology. The second is the adapter case, where compatibility can be enforced unilaterally by a single firm using an adapter. It is natural to expect that compatibility is more likely to be attained under the adapter case than the consensus case. To focus on the pure welfare implication of firms' incentives for compatibility, we assume that the cost of achieving compatibility is negligible in both cases. ${ }^{5}$

\section{Complete Cost Leadership}

We first consider the case where one firm has cost advantages in both components. Let us assume, for simplicity, that firm 1's marginal cost is $c>0$ and firm 2's marginal cost is zero for both components.

\footnotetext{
5 Adding a small cost of achieving compatibility in our model would not change the results qualitatively.
} 
Price Equilibria under Incompatibility and Compatibility. When the components produced by different firms are incompatible, the competition is system versus system and what matters to consumers in their purchasing decision is the aggregate price of a system. A consumer located at $\left(x_{1}, x_{2}\right)$ will be indifferent between the two systems if

$$
x_{1}+x_{2}+P_{1}=2-x_{1}-x_{2}+P_{2},
$$

where $P_{i}$ represents the price of firm $i$ 's system. For $P_{1} \geq P_{2}$, the firms' demands and profits are

$$
\begin{aligned}
D_{1}=\frac{1}{2}\left(1-\frac{P_{1}-P_{2}}{2}\right)^{2}, & \pi_{1}=\frac{1}{2}\left(P_{1}-2 c\right)\left(1-\frac{P_{1}-P_{2}}{2}\right)^{2}, \\
D_{2}=1-\frac{1}{2}\left(1-\frac{P_{1}-P_{2}}{2}\right)^{2}, & \pi_{2}=P_{2}\left[1-\frac{1}{2}\left(1-\frac{P_{1}-P_{2}}{2}\right)^{2}\right] .
\end{aligned}
$$

Each firm will choose its system price to maximize profits given the rival's price. The equilibrium can be easily derived from the first-order conditions.

When the components produced by the two firms are compatible with each other, the competition is component versus component. Consumers can mix and match components produced by different firms, and therefore individual consumers' choices of components are entirely independent of each other. Consumers who are indifferent between firm 1's component $A$ and firm 2's component $A$ are characterized by

$$
x_{1}+p_{A 1}=1-x_{1}+p_{A 2},
$$

and similarly for component $B$ the indifferent consumers are characterized by

$$
x_{2}+p_{B 1}=1-x_{2}+p_{B 2},
$$

where $p_{z i}$ represents the price of firm $i$ 's component $z(i=1,2, z=A, B)$. The firm $i$ 's demand for component $z$ is given by

$$
D_{z i}=\frac{1}{2}\left[1-\left(p_{z i}-p_{z j}\right)\right],
$$

and the firms' profits are

$$
\begin{gathered}
\pi_{1}=\frac{1}{2}\left(p_{A 1}-c\right)\left[1-\left(p_{A 1}-p_{A 2}\right)\right]+\frac{1}{2}\left(p_{B 1}-c\right)\left[1-\left(p_{B 1}-p_{B 2}\right)\right], \\
\pi_{2}=\frac{1}{2} p_{A 2}\left[1-\left(p_{A 2}-p_{A 1}\right)\right]+\frac{1}{2} p_{B 2}\left[1-\left(p_{B 2}-p_{B 1}\right)\right] .
\end{gathered}
$$

Each firm's maximization problem is separable in components. Focusing on the case where both firms are active in equilibrium (i.e., $c \leq 3$ ), the equilibrium is derived from the first-order conditions. We summarize the equilibrium values under the two regimes in Table 1.

Private and Social Incentives for Compatibility. Comparing the firms' profits under the two equilibria, we can find that there exists a cutoff value $c_{E}^{*}$ such that the profit of firm 2 (the efficient one) is larger under compatibility for $c<c_{E}^{*}$ and 
Table 1

Equilibrium Values for the Case of Complete Cost Leadership

\begin{tabular}{ll}
\hline Incompatibility & Compatibility \\
\hline$P_{1}^{e}=\frac{7}{4} c+\frac{1}{4}+\frac{1}{4} \sqrt{c^{2}-2 c+9}$ & $p_{z 1}^{e}=1+\frac{2 c}{3}$ \\
$P_{2}^{e}=\frac{5}{4} c-\frac{5}{4}+\frac{3}{4} \sqrt{c^{2}-2 c+9}$ & $p_{z 2}^{e}=1+\frac{c}{3}$ \\
$s_{1}=\frac{1}{32}\left(1-c+\sqrt{c^{2}-2 c+9}\right)^{2}$ & $s_{z 1}=\frac{1}{2}-\frac{c}{6}$ \\
$s_{2}=1-s_{1}$ & $s_{z 2}=1-s_{z 1}$ \\
$\pi_{1}^{I N}=\frac{1}{128}\left(1-c+\sqrt{c^{2}-2 c+9}\right)^{3}$ & $\pi_{1}^{C}=\left(1-\frac{c}{3}\right)^{2}$ \\
$\pi_{2}^{I N}=\frac{1}{32}\left(c^{2}-2 c+19\right) \sqrt{c^{2}-2 c+9}-\frac{1}{32}(c-1)\left(c^{2}-2 c-41\right)$ & $\pi_{2}^{C}=\left(1+\frac{c}{3}\right)^{2}$ \\
$C S^{I N}=V-\frac{1}{48}\left[c^{3}-3 c^{2}+69 c-19-\left(c^{2}-2 c-33\right) \sqrt{c^{2}-2 c+9}\right]$ & $C S^{C}=V-c+\frac{c^{2}}{18}-\frac{5}{2}$ \\
$W^{I N}=V-\frac{1}{12}\left[c^{3}-3 c^{2}+6 c+8-c(c-2) \sqrt{c^{2}-2 c+9}\right]$ & $W^{C}=V-c+\frac{5}{18} c^{2}-\frac{1}{2}$ \\
\hline
\end{tabular}

Note: $s_{i}$ : market shares; $C S$ : consumer surplus; $W$ : social welfare.

under incompatibility for $c>c_{E}^{*}$, and similarly for firm 1 (the inefficient one) with a different cutoff value $c_{I}^{*}$. Note that the cutoff value is lower for the efficient firm $\left(c_{E}^{*}<c_{I}^{*}\right)$. So, the two firms have a common interest in achieving compatibility when the cost asymmetry is small $\left(c<c_{E}^{*}\right)$, and in achieving incompatibility when the cost asymmetry is large $\left(c>c_{I}^{*}\right)$. When the cost asymmetry is moderate $\left(c_{E}^{*}<c<c_{I}^{*}\right)$, however, the inefficient firm favors compatibility while the efficient one is against it. This result clearly shows that the firms' incentive for compatibility can be affected by the degree of cost asymmetry between competing firms.

First note that, as pointed out by MR\&E, compatibility creates a sort of imperfect appropriation, leading to softer price competition and higher profits than does incompatibility. ${ }^{6}$ The firms' incentive to cut price tends to be smaller under compatibility, since some of the benefits from a price cut accrue to a firm's rival, while under incompatibility a price-cutting firm can capture the full benefits of its price cut. ${ }^{7}$ This imperfect-appropriation effect basically increases firms' incentive to choose compatibility.

In the presence of cost asymmetry, however, the firms' compatibility incentives are also influenced by the following factors. First, the efficient firm's cost advantage is greater under incompatibility (system competition) than under compatibility (component competition), and this allows it to get a larger market share and profit under incompatibility than under compatibility. For a given $c$, the efficient firm's

\footnotetext{
${ }^{6}$ If the firms behave as local monopolies, compatibility also increases demands by allowing consumers to mix and match their preferred components. At the moment, we focus on the case where the market is fully covered in equilibrium. We will discuss informally the cases of partial participation with this demand-shifting effect in section 7 .

7 For instance, when a firm decreases its price for component $A$, it increases the market share for its whole system under incompatibility. Under compatibility, however, a similar price cut increases its demand for component $A$ only.
} 
Figure 1

Marginal Gains from a Price Cut under Compatibility and Incompatibility

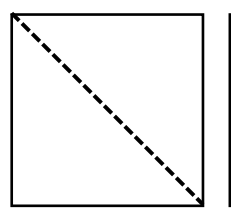

Incompatibility

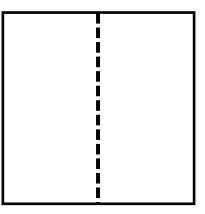

Compatibility

Symmetric Case

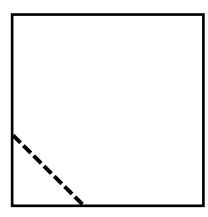

Incompatibility

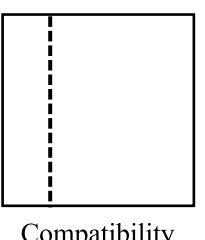

Asymmetric Case

cost advantage is $2 c$ under system competition (incompatibility), while it is reduced to $c$ under component competition (compatibility). With this asymmetry-enhancing effect the efficient firm tends to favor incompatibility, while the opposite is true for the inefficient firm. Obviously, this effect gets larger as the degree of asymmetry increases.

Second, the intensity of price competition is also affected by the gain in demand following a price cut, which is proportional to the length of the market boundary, given the distribution of consumer preferences. In the present setup with the unitsquare distribution, the equilibrium market boundary becomes shorter as the cost asymmetry increases under incompatibility, while it is independent of the degree of cost asymmetry under compatibility (see Figure 1). This market-boundary effect is reflected in the convexity of firm $i$ 's demand function $D_{i}$ in its own price under incompatibility (contrasted with the linear demand function under compatibility). Also, the corresponding competition-softening effect is confirmed by the fact that an increase in cost asymmetry tends to increase the equilibrium prices the more, the higher the degree of cost asymmetry. This leads to less intense competition under incompatibility, and its effect is increasing in the degree of cost asymmetry. Also noteworthy is that it is due to this market-boundary effect and the resulting convex demand functions that market cornering never occurs under incompatibility. Here the market boundary becomes so small that the firms' price-cutting incentive disappears at some level of cost asymmetry. With compatibility, however, the equilibrium market boundary is constant irrespective of the degree of cost asymmetry, and therefore the efficient firm corners the market for a sufficiently large degree of cost asymmetry. It should be noted that this market-boundary effect crucially depends on the distribution of consumers in the product space. For instance, for the case where consumers are distributed on a circle the equilibrium market boundary will be of equal length under compatibility and incompatibility, and therefore there is no market-boundary effect. Also, if the market-boundary effect is absent or negligible, we may have market-cornering equilibria even under incompatibility (see the analysis of the octagon distribution case in the supplementary appendix). Note that, however, the preceding imperfect-appropriation and asymmetry-enhancing effects remain valid irrespective of the consumer distribution. 
Now we can provide the economic reasoning behind the above result. When the cost asymmetry is small $\left(c<c_{E}^{*}\right)$, the asymmetry-enhancing and market-boundary effects are both negligible and the firms' price-cutting incentives are mainly affected by the imperfect-appropriation effect. So both firms prefer compatibility, as in the standard model. When the cost asymmetry is large $\left(c>c_{E}^{*}\right)$, however, the market-boundary effect is so strong that even the inefficient firm is better off with incompatibility, which yields higher equilibrium prices and profits. The efficient firm obviously favors incompatibility, on account of the asymmetry-enhancing effect. So, in this case both firms have a common interest in choosing incompatibility. Finally, when the cost asymmetry is intermediate $\left(c_{E}^{*}<c<c_{I}^{*}\right)$, the asymmetryenhancing effect exceeds the market-boundary effect, so the efficient firm prefers incompatibility, while the inefficient still favors compatibility due to the imperfectappropriation effect. So, in this case the two firms' interests conflict with regard to compatibility. ${ }^{8}$

Assuming that the first stage of the game is played sequentially - firm 2 (the efficient one) moves first, and then firm 1 (the inefficient one) follows - the equilibrium of the entire two-stage game is characterized in the following lemma. ${ }^{9}$ Obviously, in the consensus case the market outcome is determined by the efficient firm's preference for compatibility, and the opposite holds for the adapter case.

LEMMA 1 A unique perfect Nash equilibrium exists, where compatibility prevails for $c \leq c_{E}^{*}\left(c_{I}^{*}\right)$ and incompatibility prevails for $c \geq c_{E}^{*}\left(c_{I}^{*}\right)$ in the consensus (adapter) case.

We now examine the welfare implication of the market outcome. Comparing social welfare under the two equilibria leads to the following result.

LEMMA 2 There exist two cutoff values $c_{1}$ and $c_{2}\left(c_{1}<c_{E}^{*}<c_{2}<c_{I}^{*}\right)$ such that social welfare is larger under incompatibility for $c \in\left(c_{1}, c_{2}\right)$ and under compatibility for $c \in\left[0, c_{1}\right) \cup\left(c_{2}, 3\right)$.

To get some insight regarding this welfare result, it is useful to see how (in)compatibility affects the consumer gross utility (GU), average system price (AP), and total production cost (TC) (see Figure 2). First, note that compatibility, by increasing product variety, tends to increase consumer gross utility. This mix-and-match effect is very strong when the market is more or less equally divided in equilibrium (i.e., the cost asymmetry is small), yielding a larger gross utility under compatibility. This effect becomes smaller as the cost asymmetry increases, and in fact, for a sufficiently high level of cost asymmetry, consumer gross utility is larger under incompatibility, with less asymmetric market shares in equilibrium. Second, the average system

8 This possibility of firms' conflicting interests in compatibility with asymmetric costs has been pointed out by Matutes and Regibeau (1988, section 6). What is new, however, is the possibility of firms' mutual agreement on incompatibility for a sufficiently large degree of asymmetry.

9 The assumption of sequential moves allows us to sidestep the problem of multiple equilibria and the related coordination issue in the simultaneous-move game. 
price (weighted by the equilibrium market shares) tends to be higher (lower) under compatibility when the cost asymmetry is small (large). This result is a direct consequence of the relation between the relative intensity of competition under the two regimes and the degree of cost asymmetry. Third, the total production cost, a measure of production efficiency in the present setup, is closely related to the size of the inefficient firm's market share in equilibrium, which depends on the intensity of price competition as well as the degree of asymmetry. We find a nonmonotonic relationship between the relative production efficiency of the two regimes and the degree of asymmetry. When the cost asymmetry is small, production efficiency is higher under incompatibility, due to the asymmetry-enhancing effect. When the cost asymmetry is high enough, however, the market-boundary effect dominates the asymmetry-enhancing effect, leading to more efficient production under compatibility rather than incompatibility.

Note that social welfare is related to consumer gross utility and production efficiency (the price effect is canceled out). When the cost asymmetry is small $\left(c<c_{1}\right)$, the mix-and-match effect is stronger than the production-efficiency effect, leading to a larger welfare under compatibility than under incompatibility. When the cost asymmetry is moderate $\left(c_{1}<c<c_{2}\right)$, however, the production-efficiency effect dominates the mix-and-match effect, yielding the opposite result (i.e., incompatibility with more efficient production is socially desirable even though it reduces product variety). When the cost asymmetry is large $\left(c>c_{2}\right)$, the mix-and-match effect becomes insignificant and social welfare is mainly affected by the production-efficiency effect. In this case, however, production is more efficient under compatibility, with more intense price competition and smaller market shares of the less efficient firm. Also observe that consumers are better off under incompatibility (compatibility) when the cost asymmetry is small (large) (see Figure 2). This is mainly because the price effect is stronger than the mix-and-match effect.

Combining Lemmas 1 and 2, we obtain the following result on the social desirability of the market equilibrium.

PROPOSITION 1 In the consensus case, the market equilibrium involves socially excessive compatibility for $c \in\left(c_{1}, c_{E}^{*}\right)$ and socially excessive incompatibility for $c \in\left(c_{2}, 3\right)$. Similarly, in the adapter case, there is excess compatibility for $c \in\left(c_{1}, c_{2}\right)$ and excess incompatibility for $c \in\left(c_{I}^{*}, 3\right)$.

Proposition 1 clearly shows that, unlike the standard result obtained in the symmetric setup, the market incentive for compatibility may diverge from the social optimum in the system market served by asymmetric firms, regardless of the mechanism by which compatibility is attained. For instance, in the consensus case for $c_{1}<c<c_{E}^{*}$ the firms prefer compatibility, leading to softer price competition due to the imperfect-appropriation effect, but it involves too much inefficient production by the high-cost firm. Conversely, for $c>c_{2}$ the firms favor incompatibility, with less intense competition due to the market-boundary effect, while social welfare would be larger under compatibility, with more efficient production. A similar pattern emerges in the adapter case. 
Figure 2

Equilibrium Comparison between the Two Regimes $(V=10)$
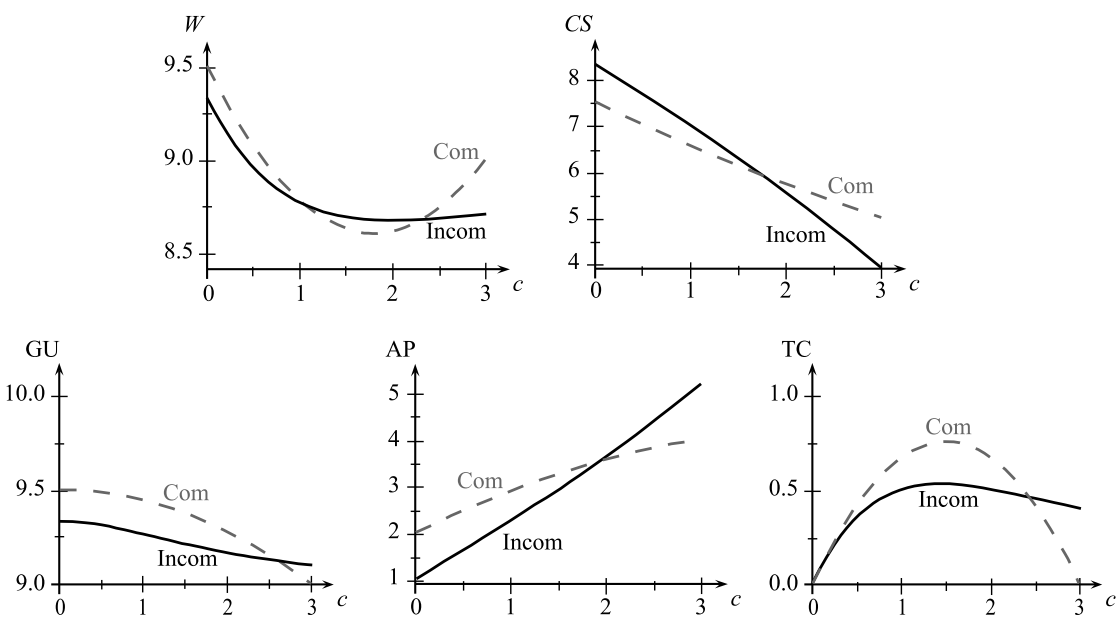

Excess incompatibility for a large degree of cost asymmetry is mainly driven by the market-boundary effect, and so the validity of this result generally depends on the distribution of consumers in the product space. ${ }^{10}$ Nevertheless we can easily see that such a result will emerge provided the consumer distribution is more concentrated in the middle under incompatibility than under compatibility, as in the present unitsquare distribution. It should be noted, however, that the excess-compatibility result is quite robust with respect to the shape of the consumer distribution, and thus deserves more attention in the arena of public policy.

\section{Shared Cost Leadership}

We now analyze the case where cost leadership is shared between the two firms across components. Suppose that firm 1 produces component $A$ more efficiently, and conversely for component $B$. It is assumed that for each firm the marginal production cost is $c>0$ for the component it produces inefficiently and zero for the one it produces efficiently.

Under compatibility, each firm's profit-maximization problem, which is separable in components, is the same as the one we analyzed in the complete-leadership case

10 The excess-incompatibility result would disappear if the consumer distribution were such that the market boundary was more or less the same for compatibility and for incompatibility, as in the case of the octagon distribution analyzed in the supplementary material. 
Figure 3

Private and Social Incentives for Compatibility

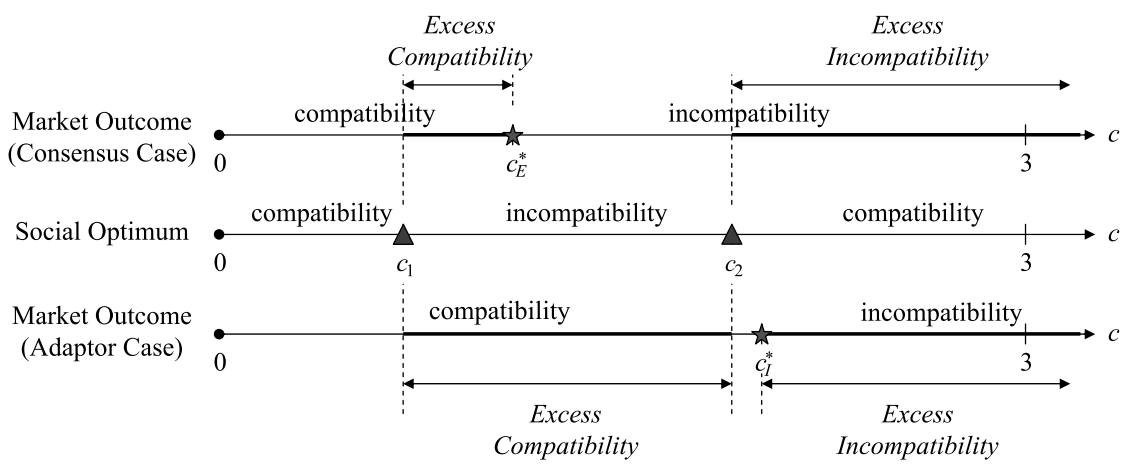

(except for changing the firm indices for component $A$ ). ${ }^{11}$ Under incompatibility, however, competition is essentially between two system providers with the same production $\operatorname{cost} c$. The equilibrium values in the two regimes are summarized in Table 2.

Table 2

Equilibrium Values for the Case of Shared Cost Leadership

\begin{tabular}{ll}
\hline Incompatibility & Compatibility \\
\hline$P_{1}^{e}=P_{2}^{e}=1+c$ & $p_{A 1}^{e}=p_{B 2}^{e}=1+\frac{c}{3}, p_{A 2}^{e}=p_{B 1}^{e}=1+\frac{2 c}{3}$ \\
$s_{1}=s_{2}=\frac{1}{2}$ & $s_{A 1}=s_{B 2}=\frac{1}{2}+\frac{c}{6}, s_{A 2}=s_{B 1}=\frac{1}{2}-\frac{c}{6}$ \\
$\pi_{1}^{I N}=\pi_{2}^{I N}=\frac{1}{2}$ & $\pi_{1}^{C}=\pi_{2}^{C}=1+\frac{c^{2}}{9}$ \\
$C S^{I N}=V-c-\frac{5}{3}$ & $C S^{C}=V-c+\frac{c^{2}}{18}-\frac{5}{2}$ \\
$W^{I N}=V-c-\frac{2}{3}$ & $W^{C}=V-c+\frac{5}{18} c^{2}-\frac{1}{2}$ \\
\hline
\end{tabular}

Comparing the two equilibria yields the following result.

PROPOSITION 2 Under shared cost leadership, the firms prefer compatibility and it is socially desirable as well. Consumers, however, are worse off under compatibility.

With shared cost leadership, the market-boundary effect vanishes, since the market boundary under incompatibility is constant at its maximum length, independent

11 Again, we focus on the case where both firms are active in equilibrium (i.e., $c \leq 3)$. 
of the degree of cost asymmetry (the two firms are essentially symmetric system suppliers). So, the firms' price-cutting incentive is affected only by the imperfectappropriation effect, and therefore the price competition will be less intense under compatibility. Then, assuming sequential plays in the first stage (either firm moves first), compatibility is clearly the unique perfect equilibrium of the two-stage game. Also worth noting is that the firms' profits under compatibility increase with the degree of cost asymmetry, while their profits under incompatibility are constant. This is because for a larger $c$ the efficient firm is less eager to capture the rival's customers, who are far from its location, and with strategic complementarity the inefficient firm also tends to raise its price. Here compatibility also increases production efficiency by allowing the firms to specialize in the component they produce efficiently. Finally, note that the asymmetry-enhancing effect is neutralized in this case of shared cost leadership.

To understand the welfare result, recall that with symmetric firms social welfare is larger under compatibility. Under shared cost leadership, compatibility is even more desirable, because it allows for efficient production. So, social welfare must be larger under compatibility than under incompatibility. This efficient production effect due to specialization dominates the mix-and-match effect as the degree of cost asymmetry increases. Consumers, however, becomes worse off under compatibility. The benefit from the mix-and-match effect is dominated by the loss from higher prices with compatibility.

\section{Independent Component Suppliers}

In this section, we examine the case where the market is served by four independent component suppliers rather than two integrated firms. Suppose that each component market is supplied by two firms producing differentiated products. We index individual firms by $z i(z=A, B, i=1,2)$, and assume that firms $A 1$ and $B 1$ are located at $(0,0)$ and firms $A 2$ and $B 2$ at $(1,1)$ on the unit square. In this setup, we investigate whether any two suppliers of complementary components have incentives to form an exclusive alliance making their components compatible only with the alliance partner. ${ }^{12}$ With only two suppliers for each component, if an alliance is formed, it immediately enforces incompatibility between the components supplied by the members of the alliance and those supplied by outsiders, leading to system competition between two integrated systems. We consider a two-stage game where the firms first decide whether to form an exclusive alliance (which is irreversible),

12 One may wonder if the firms have incentives to merge instead of forming an alliance. But a merger would be less profitable than the technological alliance, since it would reduce the joint profit of the two firms due to the internalization of complementarity. Note that, however, merger incentives may exist among three firms if the merging entity intends to monopolize the whole market by tying and foreclosing the remaining component supplier. We do not intend to explore this topic, which has been actively pursued by numerous authors (see Whinston, 1990, Choi and Stefanadis, 2001, and Carlton and Waldman, 2002, among others). 
and then compete in price independently. It is assumed that forming an alliance requires a bilateral agreement between participants.

Note that with separate ownership there is no difference in the degree of internalization of complementarity between compatibility and incompatibility, which sharply contrasts with the previous case of integrated firms. In fact, as shown by Matutes and Regibeau (1988), in the symmetric setup the price-cutting incentive is exactly the same under the compatibility and incompatibility equilibria, and therefore the firms have no incentives to form alliances. An interesting question is, however, whether the indifference result is still valid in the presence of cost asymmetry among firms. Suppose two firms supplying component $z(z=A, B)$ have different marginal costs of production: $c>0$ for the inefficient and 0 for the efficient firm. Without loss of generality, we assume that the firms located at $(0,0)$, $A 1$ and $B 1$, are inefficient and those located at $(1,1), A 2$ and $B 2$, are efficient. Note that, given the symmetric consumer preferences across components, this setup is qualitatively equivalent to the case of two asymmetric firms located at the same location; e.g., $A 1$ and $B 2$ are inefficient and $B 1$ and $A 2$ are efficient.

Equilibria under Different Alliance Structures. There are two types of alliances we need to consider. One is the case where two efficient firms $(A 2 / B 2)$ form an alliance making their components incompatible with those offered by two inefficient firms $(A 1 / B 1)$. The other is the case where two symmetric alliances are formed, each consisting of one efficient firm and one inefficient firm $(A 1 / B 2$ and $A 2 / B 1)$. It is easy to see that the latter type of alliances will not be observed in equilibrium. The efficient firms will not ally with an inefficient firm, given that their cost advantage is unilaterally passed through to the inefficient partner due to complementarity between components. Also, such alliances would intensify price competition by making two system suppliers more symmetric. So we focus on the former type of alliances.

Under the alliances, competition is between two incompatible systems. Consumers who are indifferent between the systems are characterized by

$$
x_{1}+x_{2}+p_{A 1}+p_{B 1} \leq 2-x_{1}-x_{2}+p_{A 2}+p_{B 2},
$$

where $p_{z i}$ is the component price set by firm $z i(z=A, B, i=1,2)$. For $p_{A 1}+p_{B 1} \geq$ $p_{A 2}+p_{B 2}$, the demands and profits are

$$
\begin{aligned}
& D_{A 1 / B 1}=\frac{1}{2}\left(1-\frac{p_{A 1}+p_{B 1}-\left(p_{A 2}+p_{B 2}\right)}{2}\right)^{2}, \\
& D_{A 2 / B 2}=1-\frac{1}{2}\left(1-\frac{p_{A 1}+p_{B 1}-\left(p_{A 2}+p_{B 2}\right)}{2}\right)^{2}, \\
& \pi_{z 1}=\frac{1}{2}\left(p_{z 1}-c\right)\left(1-\frac{p_{A 1}+p_{B 1}-\left(p_{A 2}+p_{B 2}\right)}{2}\right)^{2}, \\
& \pi_{z 2}=p_{z 2}\left[1-\frac{1}{2}\left(1-\frac{p_{A 1}+p_{B 1}-\left(p_{A 2}+p_{B 2}\right)}{2}\right)^{2}\right] .
\end{aligned}
$$


Table 3

Equilibrium Values for the Case of Independent Component Suppliers

\begin{tabular}{|c|c|}
\hline Alliance (incompatibility) & No alliance (compatibility) \\
\hline$p_{z 1}^{e}=\frac{5}{6} c+\frac{1}{6}+\frac{1}{6} \sqrt{c^{2}-2 c+25}$ & $p_{z 1}^{e}=1+\frac{2 c}{3}$ \\
\hline$p_{z 2}^{e}=\frac{2}{3} c-\frac{2}{3}+\frac{1}{3} \sqrt{c^{2}-2 c+25}$ & $p_{z 2}^{e}=1+\frac{c}{3}$ \\
\hline$s_{z 1}=\frac{1}{72}\left(1-c+\sqrt{c^{2}-2 c+25}\right)^{2}$ & $s_{z 1}=\frac{1}{2}-\frac{c}{6}$ \\
\hline$s_{z 2}=1-s_{z 1}$ & $s_{z 2}=1-s_{z 1}$ \\
\hline$\pi_{z 1}^{A}=\frac{1}{432}\left(1-c+\sqrt{c^{2}-2 c+25}\right)^{3}$ & $\pi_{z 1}^{N A}=\frac{1}{2}\left(1-\frac{c}{3}\right)^{2}$ \\
\hline$\pi_{z 2}^{A}=\begin{array}{l}\frac{1}{108}\left(2 c-2+\sqrt{c^{2}-2 c+25}\right) \\
\times\left(23+2 c-c^{2}+(c-1) \sqrt{c^{2}-2 c+25}\right)\end{array}$ & $\pi_{z 2}^{N A}=\frac{1}{2}\left(1+\frac{c}{3}\right)^{2}$ \\
\hline$C S^{A}=\begin{array}{l}V-\frac{1}{162}\left[c^{3}-3 c^{2}+237 c-73\right. \\
\left.-\left(c^{2}-2 c-101\right) \sqrt{c^{2}-2 c+25}\right]\end{array}$ & $C S^{N A}=V-c+\frac{c^{2}}{18}-\frac{5}{2}$ \\
\hline$W^{A}=\begin{array}{l}V-\frac{1}{162}\left[7 c^{3}-21 c^{2}+93 c+83\right. \\
\left.-\left(7 c^{2}-14 c-5\right) \sqrt{c^{2}-2 c+25}\right]\end{array}$ & $W^{N A}=V-c+\frac{5}{18} c^{2}-\frac{1}{2}$ \\
\hline
\end{tabular}

Individual firms choose their price independently to maximize profits, and the equilibrium can be easily characterized from the first-order conditions.

Without alliances, compatibility prevails and the competition is component versus component. Then, the equilibrium is exactly the same as in the previous completecost-leadership case with two integrated firms (except that an individual firm's profit is halved). ${ }^{13}$ The equilibrium values under the two regimes are summarized in Table 3.

Private and Social Incentives for Exclusive Alliances (Incompatibility). Let us now examine the firms' first-stage alliance decision. Comparing the two equilibria above, we can easily see that the alliance (incompatibility) yields larger profits for all firms $\left(\pi_{z i}^{A} \geq \pi_{z i}^{N A}\right.$ for $z=A, B, i=1,2$, where equality holds only for $c=0$ ). So, all firms will prefer alliance (incompatibility) rather than independent competition (compatibility). This is mainly because the market boundary is shorter and therefore price competition is less intense under the alliances than under independent competition. For instance, the equilibrium price of a component supplied by an inefficient firm is higher under the alliance than under independent competition, and the price difference becomes larger as the degree of cost asymmetry increases, as depicted in Figure 4.

With cost asymmetry, the efficient component suppliers have incentives to form an exclusive alliance. ${ }^{14}$

13 We assume that $c \leq 3$ to ensure no cornering in equilibrium.

14 Note that if the degree of product differentiation differs between the two components (i.e., $\lambda_{A} \neq \lambda_{B}$ ), the efficient supplier of the more differentiated component may 
Figure 4

Equilibrium Prices under Alliances and Independent Competition

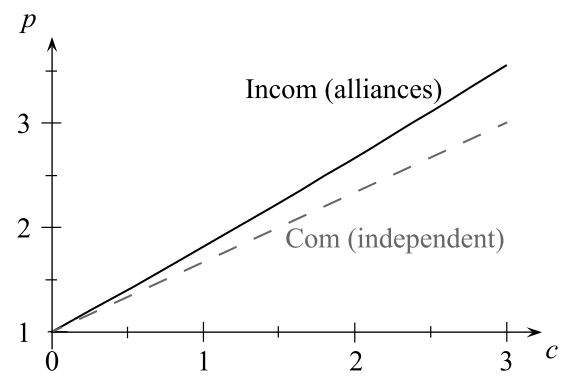

Consumers are, however, worse off under the alliances. Prices are higher under the alliances. System variety is also decreased under the alliances unless $c$ is very large (see consumer gross utility (GU) in Figure 5). Even if $c$ is large enough so that system variety is higher under the alliances, the price effect dominates the varietyincreasing effect. Total welfare is also smaller under the alliance, since it involves too little product variety when $c$ is small and too much inefficient production when $c$ is large.

Figure 5

Gross Utilities, Average System Prices, and Total Costs $(V=10)$
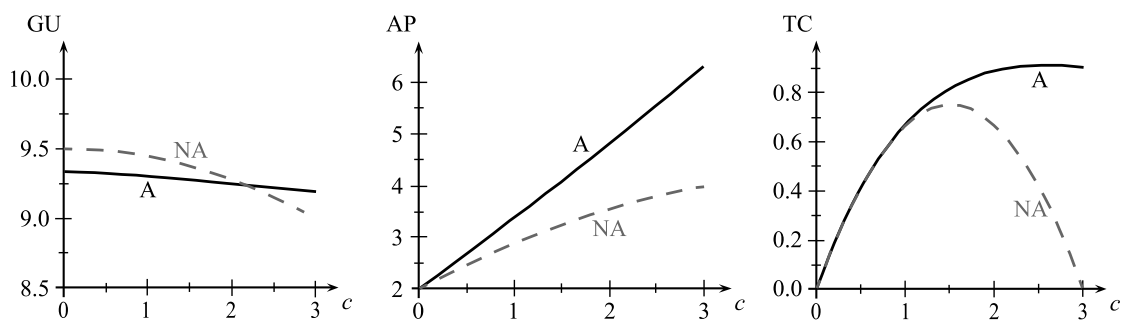

LEMMA 3 Consumer surplus and social welfare are both larger with independent competition without alliances.

PROPOSITION 3 With independent component suppliers with asymmetric costs, incompatibility prevails in the market in the form of exclusive alliances, but it is socially undesirable.

go against alliance, unless the cost asymmetry is sufficiently large. There exists a serious imperfect-appropriation problem due to complementarity under alliance. So, he would rather choose to enjoy the full benefit of the high degree of product differentiation without alliance. 
The efficient component suppliers enforce incompatibility in the market through exclusive technological alliances. This alliance structure, however, reduces social welfare by restraining consumers from mixing and matching their preferred components for small cost asymmetry, and by reducing production efficiencies for large cost asymmetry. Note that this is a new finding, which has not been addressed in the previous works.

\section{Robustness and Extensions}

We have seen that the market-boundary effect played a crucial role in part of the analysis. In particular, the validity of some results critically relied upon the shape of the consumer distribution in the product space. So, it is important to clarify which results are dependent on the assumption of the unit-square distribution, and examine how these are affected by the change of the consumer preference distribution. The most notable conclusion is that the excess-incompatibility result is sensitive to the shape of the consumer preference distribution, and will not be observed if the market boundary is the same under compatibility and incompatibility. In the supplementary appendix, we analyze the case of consumers being uniformly distributed on the regular octagon, and find that the inefficient firm always prefers compatibility, regardless of the degree of cost asymmetry, and therefore excess incompatibility never occurs even in the adapter case. ${ }^{15}$ Nevertheless, it is unfair to ignore the marketboundary effect simply because of this sort of robustness problem. On the contrary, the market-boundary effect arises whenever consumers are more concentrated in the middle under incompatibility than under compatibility. More important is the fact that this market-boundary effect is activated by asymmetries between firms, and also reinforced by the asymmetry-enhancing effect under incompatibility. Finally, we would emphasize that the excess-compatibility result is robust irrespective of the shape of the consumer distribution.

Also we need to check the robustness of our results with respect to several modeling assumptions, in particular those of consumers' full participation and unit demands. First, to examine what happens if partial participation is allowed, we need to analyze the cases of local monopolies and partial competition as in Matutes and Regibeau (1988). Unfortunately, however, it turns out that it is too complicated to fully characterize equilibria of those cases under cost asymmetry. For instance, if the market is not fully covered, the demands for different firms' components are connected to each other in a very complex way under compatibility, while those relationships become quite straightforward in a fully covered market. It is easily conjectured that with partial participation firms' incentives for compatibility tend to increase. The additional demand-shifting effect under partial participation, induced by the increased variety under compatibility, raises the firms' incentives for com-

15 Note that the octagon distribution is qualitatively similar to the unit disk and the multivariate standard normal distributions in that the market-boundary effect is neutralized under compatibility and incompatibility. 
Table 4

Equilibrium Profits under the Two Regimes for $V=2.5$

\begin{tabular}{llllc}
\hline & \multicolumn{1}{c}{$\pi_{1}^{I N}$} & \multicolumn{1}{c}{$\pi_{2}^{I N}$} & \multicolumn{1}{c}{$\pi_{1}^{C}$} & $\pi_{2}^{C}$ \\
\hline$c=0$ & 0.5 & 0.5 & 0.7763 & 0.7763 \\
$c=0.1$ & 0.4522 & 0.5863 & 0.6732 & 0.7916 \\
$c=0.2$ & 0.4085 & 0.6782 & 0.5756 & 0.8061 \\
$c=0.3$ & 0.3687 & 0.7756 & 0.4839 & 0.8195 \\
$c=0.4$ & 0.3325 & 0.8782 & 0.3986 & 0.8317 \\
$c=0.5$ & 0.2996 & 0.9858 & 0.3202 & 0.8421 \\
$c=0.6$ & 0.2698 & 1.0981 & 0.2492 & 0.8506 \\
$c=0.7$ & 0.2429 & 1.215 & 0.186 & 0.8568 \\
\hline
\end{tabular}

patibility, while the influence of the market-boundary effect becomes smaller, since the difference in the length of market boundaries between compatibility and incompatibility is less significant with partial participation than with full participation. Nevertheless, using numerical analyses, we were able to find that incompatibility may be adopted in the market provided the degree of asymmetry is sufficiently large. For example, comparing the two firms' profits under the two regimes for $V=2.5$ shows that both firms prefer compatibility for $c \leq 0.3$, the efficient firm favors incompatibility for $c \geq 0.4$, and even the inefficient firm prefers incompatibility for $c \geq 0.6$ (see Table 4). Note that here incompatibility induces direct competition, while compatibility still allows for partial participation.

Second, in order to check whether our results are robust to other demand specifications, we extended the model to the case of the same linear multiple demand structure as in Matutes and Regibeau (1988). In this setup, a consumer located at some point in the unit square is assumed to buy $X=V-s\left(d_{A i}+d_{B j}+P_{i j}\right)$ units of the system at the lowest total effective price $d_{A i}+d_{B j}+P_{i j}$. Note that with this demand specification the demand-shifting effect due to increased variety under compatibility persists even when the whole market is covered, and so it is more likely for firms to prefer compatibility. It turns out, however, that the efficient firm may still choose to make its components incompatible with the inefficient rival's for a large degree of cost asymmetry, as shown in Figure 6, which depicts the efficient (above) and inefficient (below) firms' profits as a function of $c$ for the case of $V=10$ and $s=1$. We have found qualitatively similar results for numerous examples with different values of $V$ and $s$, i.e., the efficient firm favors incompatibility for a large $c$. This shows that the asymmetry-enhancing and market-boundary effects can jointly dominate the imperfect-appropriation and demand-shifting effects with a large degree of cost asymmetry. Another interesting result is that with multiple demands, compatibility has an adverse effect on consumers and total welfare because of the double marginalization, which reduces individuals' consumption quantities. 
Figure 6

Firm Profits, Consumer Surplus, and Social Welfare for $V=10$ and $s=1$
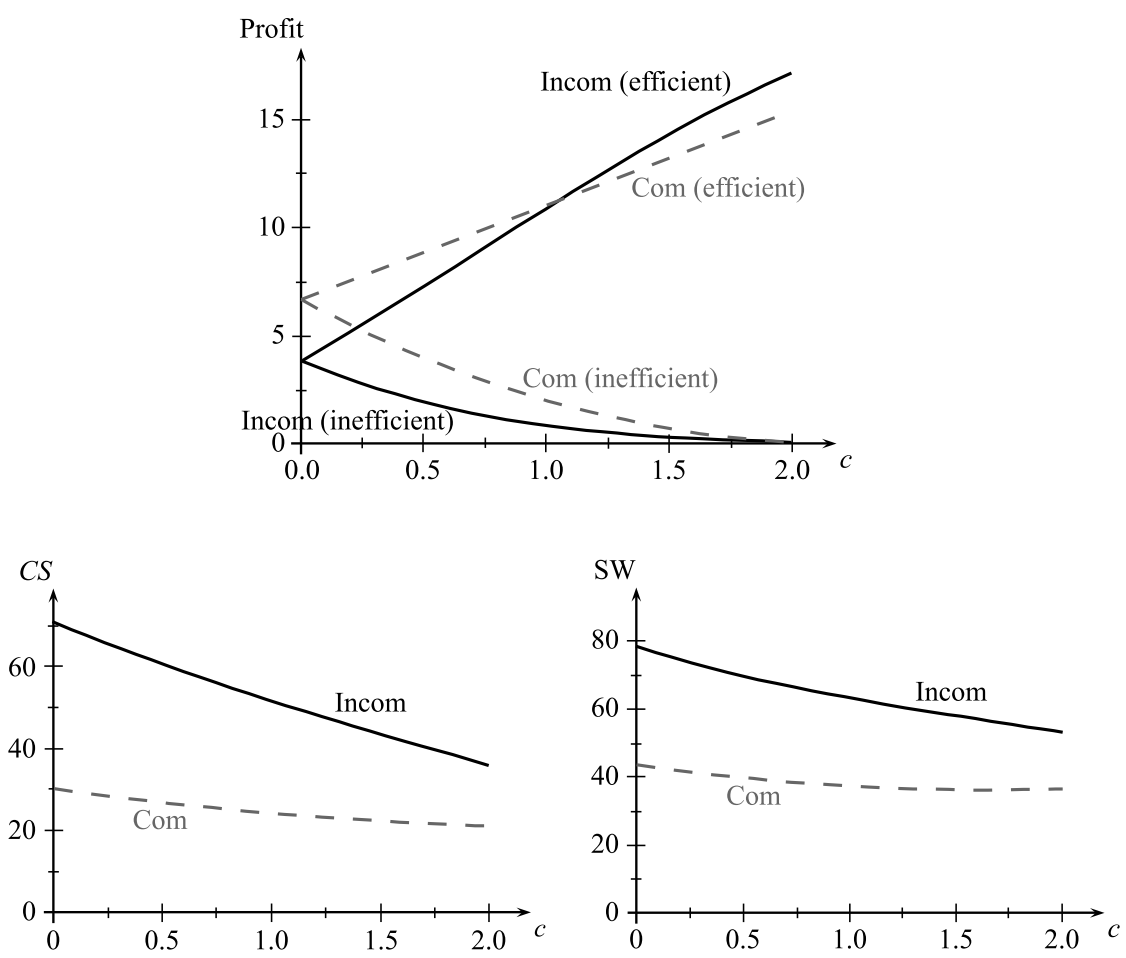

\section{Conclusion}

The early works on mix-and-match compatibility in system markets predict that compatibility prevails in the market and is also socially desirable. In this paper, we have shown that the result may change when firms competing in the market are asymmetric. In particular, if one system supplier has cost (or quality) advantages in both components (complete cost leadership), the market equilibrium tends to involve too much compatibility. The market outcome may also yield too much incompatibility if the distribution of consumer preferences is subject to the marketboundary effect and the degree of asymmetry is sufficiently large. When the market is served by independent component suppliers with different costs, incompatibility prevails in the form of exclusive technological alliances although social welfare would be higher with compatibility (i.e., no alliance formation).

Our analysis sheds new light on public policy. Competition authorities in many countries tend be lenient on (or even foster) firms' attempts to achieving compatibility in system markets. Our results cast doubt on this positive attitude, and suggest 
that policymakers need to be aware that compatibility may not be desirable when competing firms are asymmetric. Also, we need to be more suspicious of exclusive technological alliances between independent component suppliers, which tend to yield less competitive market outcomes by enforcing incompatibility among components across alliances. Overall, we need to be more careful in designing public policies in system markets, taking account of the specifics of the corresponding industry.

Given that incompatibility can be reinterpreted as bundling of two complementary products, the present analysis also offers a new perspective on product bundling and entry deterrence, which somewhat differs from the standard foreclosure argument à la Whinston (1990). In our model, incompatibility (exclusive alliances) is used as a semicollusive device rather than a foreclosing device, and so it is more closely related to the facilitating-device story where bundling relaxes competition by allowing competing firms to better differentiate themselves (see Carbajo, De Meza, and Seidman, 1990; Seidman, 1991; Chen, 1997; and Denicolo, 2000). ${ }^{16}$ This line of research usually examines the case where only one firm bundles and the other sells only one component, while we consider cases where all firms are active in equilibrium, selling their components or systems. Our result also points out the possibility that a dominant firm uses compatibility as an entry-deterring (or exit-inducing) device when entry incurs fixed costs. Since the inefficient firm's profit is smaller under compatibility than under incompatibility for an intermediate range of asymmetry, the efficient incumbent may strategically choose to enforce compatibility as a commitment to aggressive pricing in case of entry. This contrasts with the standard case where incompatibility is used as an entry-deterring device, as noted by Tirole (1988) and Nalebuff (2004). A related work on this point is that of Matutes and Regibeau (1989). They consider a setup where an incumbent firm produces one component of a two-component system and there are two incompatible versions of the second component, and show that the incumbent may wish to make its product compatible with two versions of the second component in order to limit the scope of entry in the first-component market.

\section{Supplementary Appendix}

In this appendix, we check whether the excess-compatibility and/or the excessincompatibility result still holds under circumstances where the market boundaries are similar under compatibility and incompatibility and so the market-boundary effect is neutral between the two regimes. For that purpose, we analyze the case where a continuum of consumers of mass 1 are uniformly distributed on the regular octagon of side length $l=1 / \sqrt{2(1+\sqrt{2})}$. Consumers have horizontally differentiated preferences for the components produced by the two firms. In the market

\footnotetext{
16 See also Choi (1996), who shows that bundling (incompatibility) may be used as a commitment device reducing $R \& D$ competition in asymmetric system markets.
} 
there are the two integrated system suppliers with horizontally differentiated components. Assume that firm 1's system is located at the origin and firm 2's system at $(\sqrt{(1+\sqrt{2}) / 2}, \sqrt{(1+\sqrt{2}) / 2})$ (i.e., maximal differentiation for each component).

Note that this setup is useful to evaluate the pure effect of cost asymmetry, being isolated from the bias induced by the shape of the consumer preference distribution. Unlike in the case of the unit-square distribution, the two firms face the same consumer distribution under compatibility and incompatibility, and therefore the firms' incentives are not affected by the distributional bias under this octagon distribution (see Figure A1). ${ }^{17}$

\section{Figure A1}

Densities of Market Boundary under Compatibility and Incompatibility

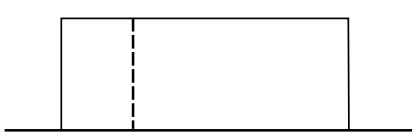

Compatibility

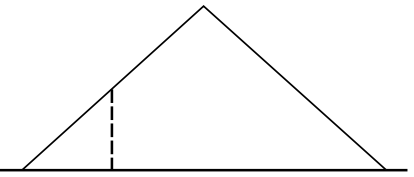

Incompatibility

Unit-Square Distribution

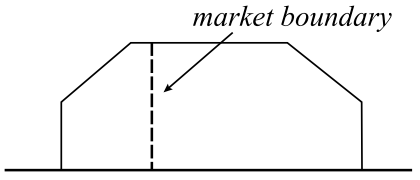

Compatibility

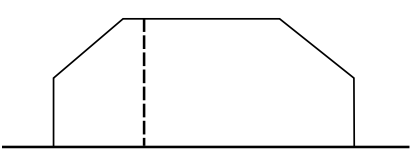

Incompatibility

Regular-Octagon Distribution

Now we characterize equilibria of the games between the two firms postulated in the main text under incompatibility and compatibility, and compare the private and social incentives for compatibility.

System Competition under Incompatibility. As we have seen in the main text, when the components produced by different firms are incompatible, the competition is system versus system, and what matters to consumers in their purchasing decision is the aggregate price of a system. A consumer located at $\left(x_{A}, x_{B}\right)$ will be indifferent

17 Alternatively, one may assume that consumers are distributed on the unit disk, which has exactly the same property as the regular octagon in that the market boundary does not change with the choice of compatibility or incompatibility. Unfortunately, however, solving for equilibria in this case is technically demanding. We strongly believe the qualitative results would not change with this distributional modification. 
between two systems if

$$
x_{A}+x_{B}+P_{1}=\sqrt{2(1+\sqrt{2})}-x_{A}-x_{B}+P_{2},
$$

where $P_{i}$ represents the price of firm $i$ 's system (see Figure A2).

We need to consider two cases, depending on the difference in the system prices offered by the two firms, due to the kink in the octagon-shaped distribution. Each firm's demand is given by

$$
\begin{aligned}
& D_{1}\left(P_{1}, P_{2}\right)=\frac{1}{2}\left[1-\frac{\sqrt{1+\sqrt{2}}}{2}\left(P_{1}-P_{2}\right)\right], \\
& D_{2}\left(P_{1}, P_{2}\right)=\frac{1}{2}\left[1-\frac{\sqrt{1+\sqrt{2}}}{2}\left(P_{2}-P_{1}\right)\right]
\end{aligned}
$$

if the price differential is small, and

$$
\begin{aligned}
& D_{1}\left(P_{1}, P_{2}\right)=\frac{1}{4}\left[\sqrt{1+\sqrt{2}}-\frac{P_{1}-P_{2}}{\sqrt{2}}\right]^{2}-\frac{\sqrt{2}-1}{8}, \\
& D_{2}\left(P_{1}, P_{2}\right)=\frac{7+\sqrt{2}}{8}-\frac{1}{4}\left[\sqrt{1+\sqrt{2}}-\frac{P_{1}-P_{2}}{\sqrt{2}}\right]^{2}
\end{aligned}
$$

if it is large.

Solving the profit-maximization problem of each firm, the first-order conditions leads to the following equilibrium prices and profits:

$$
\begin{array}{ll}
P_{1}^{e}=2 \sqrt{\sqrt{2}-1}+\frac{4}{3} c, & \pi_{1}^{I N}=\frac{(3-\sqrt{1+\sqrt{2}} c)^{2}}{9 \sqrt{1+\sqrt{2}}} \\
P_{2}^{e}=2 \sqrt{\sqrt{2}-1}+\frac{2}{3} c, & \pi_{2}^{I N}=\frac{(3+\sqrt{1+\sqrt{2}} c)^{2}}{9 \sqrt{1+\sqrt{2}}}
\end{array}
$$

for $c \leq 3 /(2 \sqrt{\sqrt{2}+1}) \simeq 0.9654$, and

$$
\begin{gathered}
P_{1}^{e}=\frac{\sqrt{2 \sqrt{2}+2}(8 \sqrt{2}-3)}{56}-\frac{(8 \sqrt{2}-59) c}{28}+\frac{(57 \sqrt{2}-32) \Phi}{6664}, \\
P_{2}^{e}=\frac{(16-45 \sqrt{2}) \sqrt{\sqrt{2}+1}+(90-16 \sqrt{2}) c+\sqrt{2} \Phi}{56}, \\
\pi_{1}^{I N}=\left(P_{1}^{e}-2 c\right)\left[\left(\frac{833 \sqrt{\sqrt{2}+1}-833 \sqrt{2} c+(31+8 \sqrt{2}) \Phi}{6664}\right)^{2}-\frac{\sqrt{2}-1}{8}\right], \\
\pi_{2}^{I N}=P_{2}^{e}\left[1+\frac{\sqrt{2}-1}{8}-\left(\frac{833 \sqrt{\sqrt{2}+1}-833 \sqrt{2} c+(31+8 \sqrt{2}) \Phi}{6664}\right)^{2}\right]
\end{gathered}
$$

for

$$
3 /(2 \sqrt{\sqrt{2}+1}) \leq c \leq \frac{1}{2}(\sqrt{2 \sqrt{2}+2}+\sqrt{17 \sqrt{2}+7}) \simeq 3.8844
$$


where

$$
\Phi=\left[(2178-992 \sqrt{2}) c^{2}-(2178 \sqrt{2}-1984) \sqrt{\sqrt{2}+1} c+9197-1003 \sqrt{2}\right]^{\frac{1}{2}} .
$$

Component Competition with Compatibility. When the components produced by the two firms are compatible with each other, the competition is component versus component. Consumers who are indifferent between the two firms' components are characterized by

$$
x_{z}+p_{z 1}=\sqrt{\frac{1+\sqrt{2}}{2}}-x_{z}+p_{z 2},
$$

where $p_{z i}$ represents the price of firm $i$ 's component $z(i=1,2, z=A, B)$ (see Figure A2).

We consider two cases, depending on the difference in the two firms' component prices. Each firm's demand for component $z$ is

$$
\begin{aligned}
& D_{z 1}\left(p_{z 1}, p_{z 2}\right)=\frac{1}{2}\left[1-\sqrt{\frac{1+\sqrt{2}}{2}}\left(p_{z 1}-p_{z 2}\right)\right], \\
& D_{z 2}\left(p_{z 1}, p_{z 2}\right)=\frac{1}{2}\left[1-\sqrt{\frac{1+\sqrt{2}}{2}}\left(p_{z 2}-p_{z 1}\right)\right]
\end{aligned}
$$

Figure A2

Equilibrium Market Configurations under Compatibility and Incompatibility
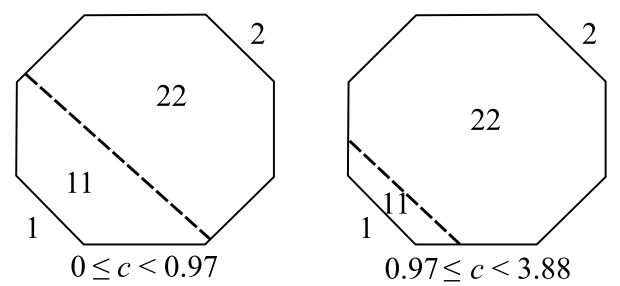

Incompatibility
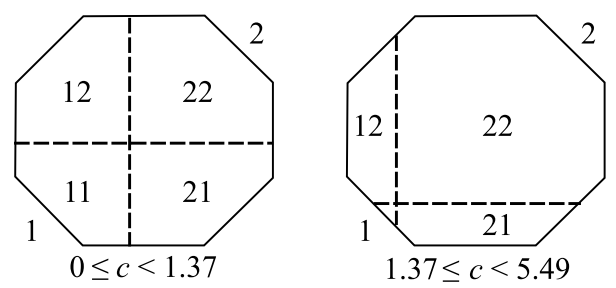

Compatibility 
if the price differential is small, and

$$
\begin{aligned}
& D_{z 1}\left(p_{z i}, p_{z j}\right)=\left[\frac{\sqrt{1+\sqrt{2}}}{2}-\frac{p_{z 1}-p_{z 2}}{2}\right]^{2}-\frac{\sqrt{2}-1}{8}, \\
& D_{z 2}\left(p_{z i}, p_{z j}\right)=\frac{7+\sqrt{2}}{8}-\left[\frac{\sqrt{1+\sqrt{2}}}{2}-\frac{p_{z 1}-p_{z 2}}{2}\right]^{2}
\end{aligned}
$$

if it is large.

Solving the profit-maximization problems, the equilibrium prices and profits are given as

$$
\begin{array}{ll}
p_{z 1}^{e}=\sqrt{2 \sqrt{2}-2}+\frac{2 c}{3}, & \pi_{1}^{C}=\frac{(6-\sqrt{2 \sqrt{2}+2} c)^{2}}{18 \sqrt{2 \sqrt{2}+2}}, \\
p_{z 2}^{e}=\sqrt{2 \sqrt{2}-2}+\frac{c}{3}, & \pi_{2}^{C}=\frac{(6+\sqrt{2 \sqrt{2}+2} c)^{2}}{18 \sqrt{2 \sqrt{2}+2}}
\end{array}
$$

for

$$
c \leq 6 \sqrt{(\sqrt{2}+1) /(4 \sqrt{2}+6)}-3 \sqrt{1 /(2 \sqrt{2}+2)} \simeq 1.3653
$$

and

$$
\begin{gathered}
p_{z 1}^{e}=\frac{(8 \sqrt{2}-3) \sqrt{\sqrt{2}+1}-\frac{(8 \sqrt{2}-59) c}{56}+\frac{(57-16 \sqrt{2}) \Omega}{6664},}{p_{z 2}^{e}=\frac{(8 \sqrt{2}-45) \sqrt{\sqrt{2}+1}+(45-8 \sqrt{2}) c+\Omega}{56},} \\
\pi_{1}^{C}=2\left(p_{z 1}^{e}-c\right)\left[\left(\frac{833 \sqrt{\sqrt{2}+1}-833 c+(31+8 \sqrt{2}) \Omega}{6664}\right)^{2}-\frac{\sqrt{2}-1}{8}\right], \\
\pi_{2}^{C}=2 p_{z 2}^{e}\left[1+\frac{\sqrt{2}-1}{8}-\left(\frac{833 \sqrt{\sqrt{2}+1}-833 c+(31+8 \sqrt{2}) \Omega}{6664}\right)^{2}\right]
\end{gathered}
$$

for

$$
\begin{aligned}
& 6 \sqrt{(\sqrt{2}+1) /(4 \sqrt{2}+6)}-3 \sqrt{1 /(2 \sqrt{2}+2)} \leq c \leq \sqrt{7 / 2+17 / \sqrt{2}}+\sqrt{\sqrt{2}+1} \\
& \simeq 5.4934
\end{aligned}
$$

where

$$
\Omega=\left[(1089-496 \sqrt{2}) c^{2}-(2178-992 \sqrt{2}) \sqrt{\sqrt{2}+1} c+9197-1003 \sqrt{2}\right]^{\frac{1}{2}} .
$$

Firms' Incentive for Compatibility. Comparing the firms' equilibrium profits under compatibility and incompatibility, we have the following result. In order to focus on the situations where both firms are active in the market, we confine our analysis to the case of $c<3.8844$. 
LEMMA A1 The efficient firm (firm 2) prefers compatibility for $c<c^{*} \simeq 1.78$ and incompatibility for $c>c^{*}$, while the inefficient firm (firm 1) always prefers compatibility to incompatibility.

Not surprisingly, the firms have a common interest in achieving compatibility when the cost differential is sufficiently small $\left(c<c^{*}\right)$. When the degree of cost asymmetry is sufficiently large $\left(c>c^{*}\right)$, however, the efficient firm prefers incompatibility while the inefficient still prefers compatibility. Note that without the market-boundary effect the firms' incentives for compatibility are solely determined by the interplay of the imperfect-appropriation and asymmetry-enhancing effects. As shown in Figure A3, when the degree of asymmetry is large $\left(c>c^{*}\right)$, the asymmetry-enhancing effect is stronger than the imperfect-appropriation effect, inducing the efficient firm to opt for incompatibility.

\section{Figure A3}

Each Firm's Profit Gain Due to Compatibility Relative to Incompatibility $\left(\pi_{i}^{C}-\pi_{i}^{I N}\right)$

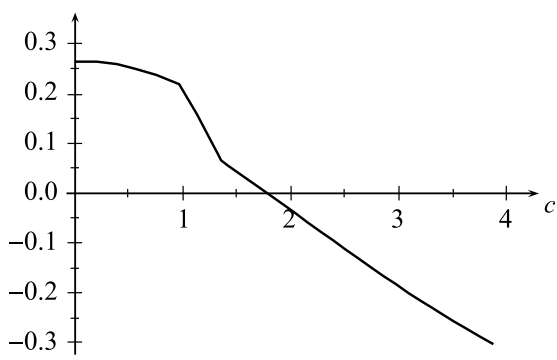

Efficient Firm

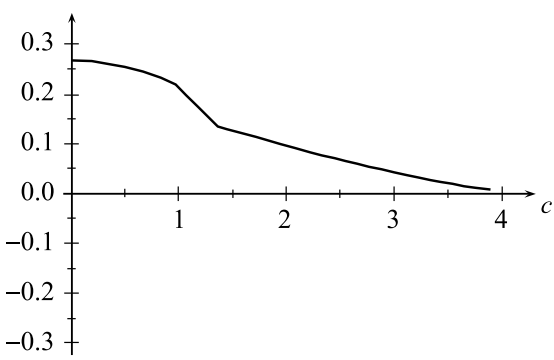

Inefficient Firm

Social Desirability for Compatibility. Using the equilibrium prices and profits obtained above, we can calculate consumer surplus and total welfare under incompatibility and compatibility as follows:

$$
\begin{aligned}
& C S^{I N}=\left\{\begin{array}{cc}
V-2.0464-c+0.0863 c^{2} & \text { for } c \leq 0.9654, \\
V+0.3961-1.3901 c+0.0687 c^{2}-0.0208 c^{3} & \\
+\left(0.0208 c^{2}-0.0458 c-0.6578\right) \Gamma_{1} & \text { for } 0.9654 \leq c \leq 3.8844,
\end{array}\right. \\
& W^{I N}=\left\{\begin{array}{cc}
V-0.7593-c+0.4316 c^{2} & \text { for } c \leq 0.9654, \\
V-0.7767-0.4741 c+0.2747 c^{2}-0.0833 c^{3} & \\
+\left(0.0833 c^{2}-0.1831 c+0.0086\right) \Gamma_{1} & \text { for } 0.9654 \leq c \leq 3.8844,
\end{array}\right. \\
& C S^{C}=\left\{\begin{array}{cc}
V-2.4390-c+0.0610 c^{2} & \text { for } c \leq 1.3653, \\
V+1.0152-1.3906 c+0.0486 c^{2}-0.0104 c^{3} & \\
+\left(0.0104 c^{2}-0.0324 c-0.6578\right) \Gamma_{2} & \text { for } 1.3653 \leq c \leq 5.4934,
\end{array}\right.
\end{aligned}
$$




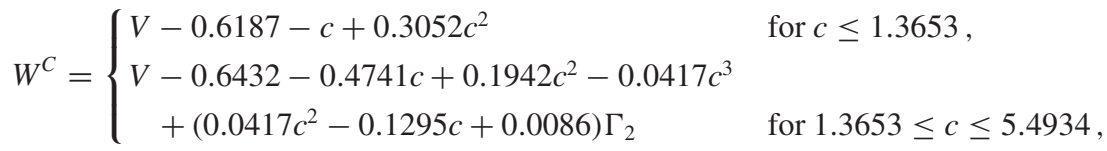

where $\Gamma_{1}=\sqrt{10.0355-2.1974 c+c^{2}}$ and $\Gamma_{2}=\sqrt{20.0711-3.1076 c+c^{2}}$.

Comparing social welfare under the two regimes leads to the following result.

LEMMA A2 Social welfare is larger under compatibility for $c<c^{s} \simeq 1.1$, and under incompatibility for $c>c^{s}$.

Figure A4

Consumer Surplus and Total Welfare under Incompatibility and Compatibility $(V=10)$
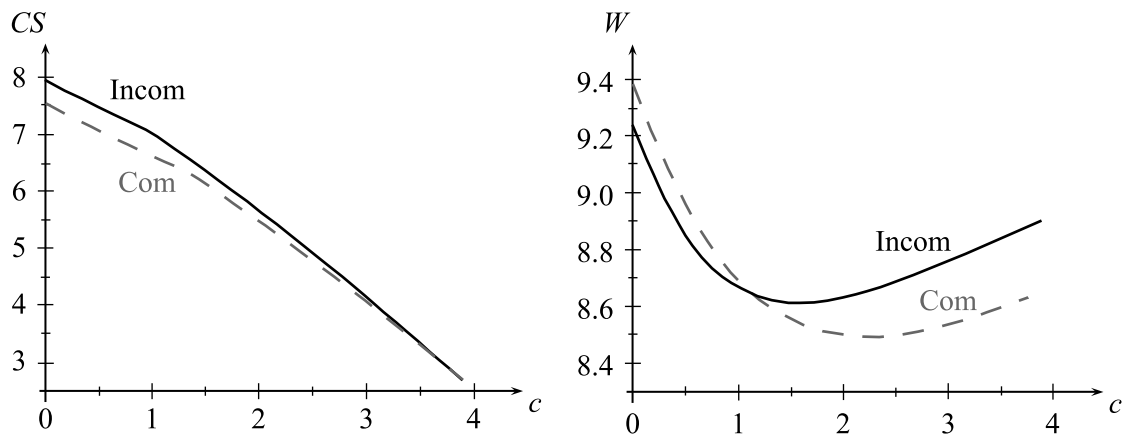

In order to get some insight into this result, it is useful to decompose the total welfare into consumer gross utility (GU), average system price (AP), and total production cost (TC) (see Figure A5). First, compatibility, by allowing mixing and matching, reduces consumers' transportation costs and leads to larger gross utilities. This mix-and-match effect is stronger when the market is more equally divided in equilibrium (i.e., the cost asymmetry is small), and gets smaller as the degree of cost asymmetry increases. Second, the average system price (weighted by the equilibrium market shares) tends to be higher under compatibility, due to the imperfect-appropriation effect. Third, given a fully covered market, production efficiency, measured by the total production cost, is solely related to how the total production is allocated between the firms in equilibrium. As noted earlier, the efficient firm enjoys a larger market share under incompatibility than under compatibility, and more importantly, its relative market share increases as the cost asymmetry increases. So, production efficiency tends to be higher under incompatibility than under compatibility.

System Variety versus Production Efficiency. Let us consider the two-stage game where each firm first decides whether to make its components compatible with its rival's, and the two then compete in price, given the first-stage compatibility 
Figure A5

Consumer Gross Utility $(V=10)$, Average System Price, and Total Production Cost
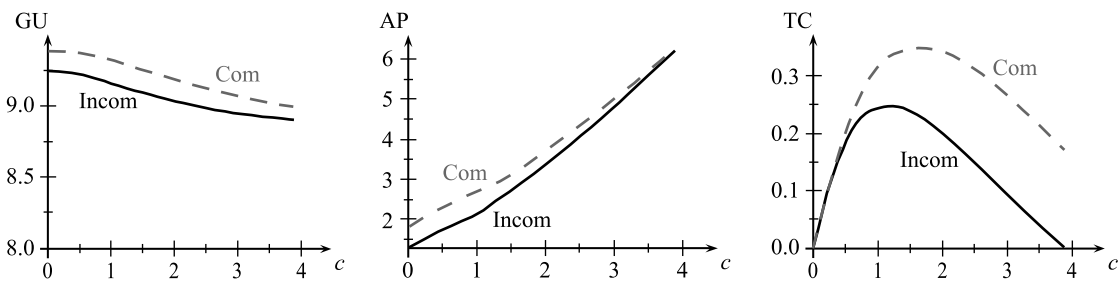

decisions. The first-stage compatibility choices are assumed to be irreversible. As before, we consider two mechanisms by which compatibility is attained in the market, viz., the consensus case and the adapter case. Solving for a perfect Nash equilibrium, we obtain the following result, which is immediate from Lemma A1.

LEMMA A3 In the unique perfect Nash equilibrium of the two-stage game, (i) compatibility (incompatibility) is achieved for $c \leq c^{*} \simeq 1.78\left(c \geq c^{*}\right)$ in the consensus case, and (ii) compatibility always prevails in the adapter case.

Combining Lemmas A2 and A3 leads to the following proposition on the social desirability of the market equilibrium.

PROPOSITION A The market equilibrium involves socially excessive compatibility for $c \in\left(c^{s}, c^{*}\right)$ in the consensus case, and for $c \in\left(c^{s}, \infty\right)$ in the adapter case.

This result clearly shows that excess compatibility can happen in system markets even without the market-boundary effect. So, the market incentive for compatibility can diverge from the social optimum, regardless of the consumers' taste distribution and the mechanism by which compatibility is attained.

Figure A6

Private and Social Incentives for Compatibility

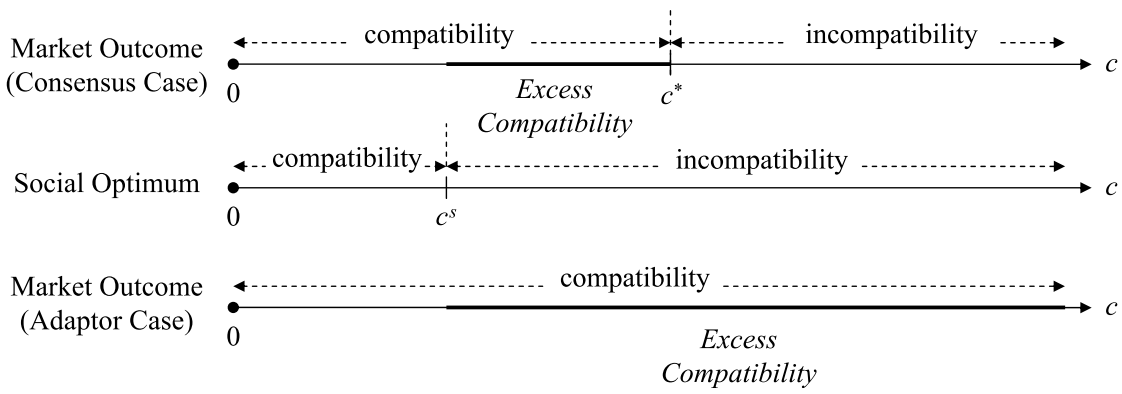




\section{References}

Boom, A. (2001), "On the Desirability of Compatibility with Product Selection," The Journal of Industrial Economics, 49, 85-96.

Carbajo, J., D. De Meza, and D. Seidman (1990), "A Strategic Motivation for Commodity Bundling," The Journal of Industrial Economics, 38, 283-289.

Carlton, D., and M. Waldman (2002), "The Strategic Use of Tying to Preserve and Create Market Power in Evolving Industries," The RAND Journal of Economics, 33, 194-220.

Chen, Y. (1997), "Equilibrium Product Bundling," The Journal of Business, 70, 85-103.

Choi, J. (1996), "Preemptive R\&D, Rent Dissipation, and the 'Leverage theory'," The Quarterly Journal of Economics, 111, 1153-1181.

— and C. Stefanadis (2001), "Tying, Investment and the Dynamic Leverage Theory," The RAND Journal of Economics, 32, 52-71.

Denicolo, V. (2000), "Compatibility and Bundling with Generalist and Specialist Firms," The Journal of Industrial Economics, 48, 177-188.

Economides, N. (1989), "Desirability of Compatibility in the Absence of Network Externalities," The American Economic Review, 79, 1165-1181.

Einhorn, M. (1992), "Mix and Match Compatibility with Vertical Product Dimensions," The RAND Journal of Economics, 23, 535-547.

Farrell, J., H. Monroe, and G. Saloner (1998), “The Vertical Organization of Industry: Systems Competition versus Component Competition," Journal of Economics \& Management Strategy, 7, 143-182.

Matutes, C., and P. Regibeau (1988), “Mix and Match”: Product Compatibility without Network Externalities," The RAND Journal of Economics, 19, 221-234.

— and - (1989), "Standardization across Markets and Entry," The Journal of Industrial Economics, 37, 357-371.

— and - (1992), "Compatibility and Bundling of Complementary Goods in a Duopoly," The Journal of Industrial Economics, 40, 37-54.

Nalebuff, B. (2004), "Bundling as an Entry Barrier," The Quarterly Journal of Economics, $119,159-187$.

Seidman, D. (1991), "Bundling as a Facilitating Device: A Reinterpretation of Leverage Theory," Economica, 58, 491-499.

Tirole, J. (1988), The Theory of Industrial Organization, The MIT Press, Cambridge (MA).

Whinston, M. (1990), "Tying, Foreclosure and Exclusion," The American Economic Review, $80,837-859$.

Jong-Hee Hahn

School of Economics

Yonsei University

Yonsei-ro, Seodaemun-gu

Seoul 120-749

Korea

E-mail:

hahnjh@yonsei.ac.kr
Sang-Hyun Kim

Department of Economics

Michigan State University

110 Marshall-Adams Hall

East Lansing, MI 48824

U.S.A.

E-mail:

kimsan46@gmail.com 